\title{
Short intronic repeat sequences facilitate circular RNA production
}

\author{
Dongming Liang and Jeremy E. Wilusz \\ Department of Biochemistry and Biophysics, University of Pennsylvania Perelman School of Medicine, Philadelphia, \\ Pennsylvania 19104, USA
}

\begin{abstract}
Recent deep sequencing studies have revealed thousands of circular noncoding RNAs generated from proteincoding genes. These RNAs are produced when the precursor messenger RNA (pre-mRNA) splicing machinery "backsplices" and covalently joins, for example, the two ends of a single exon. However, the mechanism by which the spliceosome selects only certain exons to circularize is largely unknown. Using extensive mutagenesis of expression plasmids, we show that miniature introns containing the splice sites along with short ( 30- to 40nucleotide) inverted repeats, such as Alu elements, are sufficient to allow the intervening exons to circularize in cells. The intronic repeats must base-pair to one another, thereby bringing the splice sites into close proximity to each other. More than simple thermodynamics is clearly at play, however, as not all repeats support circularization, and increasing the stability of the hairpin between the repeats can sometimes inhibit circular RNA biogenesis. The intronic repeats and exonic sequences must collaborate with one another, and a functional $3^{\prime}$ end processing signal is required, suggesting that circularization may occur post-transcriptionally. These results suggest detailed and generalizable models that explain how the splicing machinery determines whether to produce a circular noncoding RNA or a linear mRNA.
\end{abstract}

[Keywords: ZKSCAN1; HIPK3; EPHB4; splicing; noncoding RNA; Alu; circRNA]

Supplemental material is available for this article.

Received August 27, 2014; revised version accepted September 18, 2014.

Most of the eukaryotic genome is transcribed, yielding a complex repertoire of transcripts that includes tens of thousands of individual noncoding RNAs with little or no predicted protein-coding capacity (for review, see Wilusz et al. 2009; Cech and Steitz 2014; Yang et al. 2014). Although relatively few noncoding RNAs have been assigned a function, their existence is a challenge to the long-held assumption that most genetic information is expressed as and carried out by proteins. Unexpectedly, even protein-coding regions of the genome-including genes with highly evolutionarily conserved ORFsgenerate noncoding RNAs. Unlike linear messenger RNAs (mRNAs), these noncoding RNAs often have covalently linked ends, making them circles (for review, see Wilusz and Sharp 2013; Jeck and Sharpless 2014). A handful of such circular RNAs were serendipitously found over the past 25 years (Nigro et al. 1991; Capel et al. 1993; Cocquerelle et al. 1993; Zaphiropoulos 1997; Burd et al. 2010), but it is becoming increasingly clear that this class of noncoding RNA is much larger than previously appreciated (Salzman et al. 2012). Over 25,000 circular RNAs, derived from $\sim 15 \%$ of actively tran-

Corresponding author: wilusz@mail.med.upenn.edu Article published online ahead of print. Article and publication date are online at http://www.genesdev.org/cgi/doi/10.1101/gad.251926.114. scribed genes, were recently identified in a single human cell type (Jeck et al. 2013). These highly stable transcripts contain almost exclusively exonic sequences, localize to the cytoplasm, and, at least in two cases, function as efficient modulators of microRNA activity (Hansen et al. 2013; Memczak et al. 2013). Thousands of circular RNAs have additionally been found in numerous other eukaryotes (Salzman et al. 2012; Memczak et al. 2013; Guo et al. 2014; Wang et al. 2014) as well as in archaea (Danan et al. 2012). For some genes, the abundance of the circular RNA exceeds that of the associated linear mRNA by a factor of 10 , raising the interesting possibility that the function of some protein-coding genes may actually be to produce circular noncoding RNAs, not proteins.

Because eukaryotes contain split, intron-containing genes, their precursor mRNAs (pre-mRNAs) must be modified such that introns are removed and exons are joined together. If a pre-mRNA is spliced in the standard way (meaning exon 1 is joined to exon 2, which is joined to exon 3, etc.), a linear mRNA is generated that can be

(C) 2014 Liang and Wilusz This article is distributed exclusively by Cold Spring Harbor Laboratory Press for the first six months after the full-issue publication date (see http://genesdev.cshlp.org/site/misc/terms.xhtml). After six months, it is available under a Creative Commons License (Attribution-NonCommercial 4.0 International), as described at http:// creativecommons.org/licenses/by-nc/4.0/. 
subsequently translated to produce a protein. Alternatively, the splicing machinery can "backsplice" and join a splice donor to an upstream splice acceptor, thereby yielding a circular RNA that has covalently linked ends. Supporting this biogenesis model, canonical splicing signals generally immediately flank regions that circularize, and exon circularization can be observed when splicing substrates are incubated in mammalian nuclear extracts (Braun et al. 1996; Pasman et al. 1996; Schindewolf et al. 1996). Once generated, circular RNAs appear to almost always be noncoding, as the genic start and/or stop codons have been removed. Although artificial circular RNAs containing an internal ribosome entry site (IRES) can be translated in cellular extracts (Chen and Sarnow 1995), endogenous circular RNAs have not been found to associate with ribosomes in cells (Jeck et al. 2013; Guo et al. 2014). Therefore, the choice between production of a linear mRNA and a circular RNA must be tightly regulated, as it fundamentally changes the functional output of a gene.

With the exception of the first and last exons of genes, every other exon in the genome has splicing signals at its $5^{\prime}$ and $3^{\prime}$ ends and theoretically can circularize. However, every exon does not circularize, and, in some cases, multiple exons are present in a circular RNA. So how does the spliceosome determine whether a region of a premRNA yields a circle? The kinetics of splicing likely provides a partial answer because introns are generally removed as the gene is still being transcribed (i.e., cotranscriptionally) (for review, see Brugiolo et al. 2013). To allow sufficient time for a backsplice to occur, the intron immediately preceding the circularizing exons likely must be spliced slower than the average intron. Sequence-specific elements may additionally be required for circularization, as introns flanking exons that circularize commonly contain repeat sequences (particularly Alu elements) in an inverted orientation (Jeck et al. 2013). These repeats may base-pair, bringing the splice site sequences into close proximity to each other and facilitating catalysis. Inverted repeats of $>15 \mathrm{~kb}$ surround the mouse Sry exon that circularizes, and deletion analyses have suggested that at least 400 complementary nucleotides are necessary for Sry circularization in vivo (Dubin et al. 1995). Artificially surrounding an exon with introns containing 800 nucleotides (nt) of perfectly complementary repeats likewise is sufficient to allow circularization (Hansen et al. 2013). However, it is unclear whether the repeat length rules defined for Sry or these artificial expression vectors apply to most genes that circularize. This is because Alu elements, which are only $\sim 300 \mathrm{nt}$ in length, flank many exons that naturally generate circles. Detailed mechanistic models for how circularization generally occurs are therefore currently lacking, limiting our understanding of how circular RNAs are regulated and function.

To reveal the mechanism by which circular RNAs are produced, the pre-mRNAs of the human ZKSCAN1 (zinc finger protein with KRAB and SCAN domains 1), HIPK3 (homeodomain-interacting protein kinase 3), and EPHB4 (ephrin type B receptor 4) genes were cloned into easily manipulatable expression vectors. By extensively mutagenizing these vectors, we identified minimal regions that are sufficient for circular RNA production in cells. Surprisingly, miniature introns ( $<100 \mathrm{nt}$ ) containing only the splice site sequences along with short inverted repeats $(\sim 30-40 \mathrm{nt})$ were sufficient to allow the intervening exons to efficiently circularize. The repeats must base-pair to one another; however, more than simple thermodynamics is clearly at play, as not all intronic repeat sequences support circularization. In fact, for all three genes tested, strengthening the hairpin between the repeats sometimes strongly inhibited circularization. Within the repeat sequences, we found that G-U wobble base pairs as well as poly(A) stretches can limit circular RNA production. We further show that circularization requires a functional $3^{\prime}$ end processing signal as well as collaboration between the intronic repeats and the exonic sequences. These results suggest a generalizable model for how the splicing machinery determines whether to produce a circular RNA or a linear mRNA.

\section{Results}

The human ZKSCAN1 gene produces an abundant circular RNA

As a model to study the mechanism by which circular RNAs are produced from protein-coding genes, we first chose to focus on the human ZKSCAN1 gene. The ZKSCAN1 protein is overexpressed in certain gastroesophageal cancers (van Dekken et al. 2009) and has been suggested to regulate GABA type A receptor expression in the brain (Mulligan et al. 2012). Deep sequencing of the transcriptomes of numerous human cell lines, including HEK293, HeLa, and H1 human embryonic stem cells (hESCs), revealed exon-exon junction reads connecting the $3^{\prime}$ end of exon 3 to the $5^{\prime}$ end of exon 2 (Salzman et al. 2012; Jeck et al. 2013; Memczak et al. 2013). This suggests that exons 2 and 3 of ZKSCAN1 may be spliced together to form a covalently linked 668-nt circular RNA (Fig. 1A). Using Northern blots and an oligo probe complementary to exon 2 of ZKSCAN1, we detected an abundant transcript of the appropriate size for the circular RNA in normal human brain and liver tissues (Fig. 1B) as well as in the Huh7 human hepatoma cell line (Fig. 1C, lane 1). In Huh7 cells, the ZKSCAN1 mRNA is approximately threefold more abundant than the circular RNA. However, the circular RNA is considerably more stable (half-life of $>6$ $\mathrm{h}$ compared with $\sim 2 \mathrm{~h}$ for the linear mRNA) as determined by inhibition of transcription via flavopiridol, an elongation inhibitor (Fig. 1C; Supplemental Fig. 1A).

ZKSCAN1 circular RNA expression was further measured using quantitative PCR (qPCR) after reverse transcription on RNAs derived from an independent set of tissue donors (Supplemental Fig. 1B). The highest circular RNA levels were present in the brain, liver, skeletal muscle, and salivary gland, although the transcript was detectable in all tissues examined. Consistent with previous reports (Salzman et al. 2013; Guo et al. 2014), there was no clear correlation between ZKSCAN1 mRNA and circular RNA levels, suggesting that the higher circular 

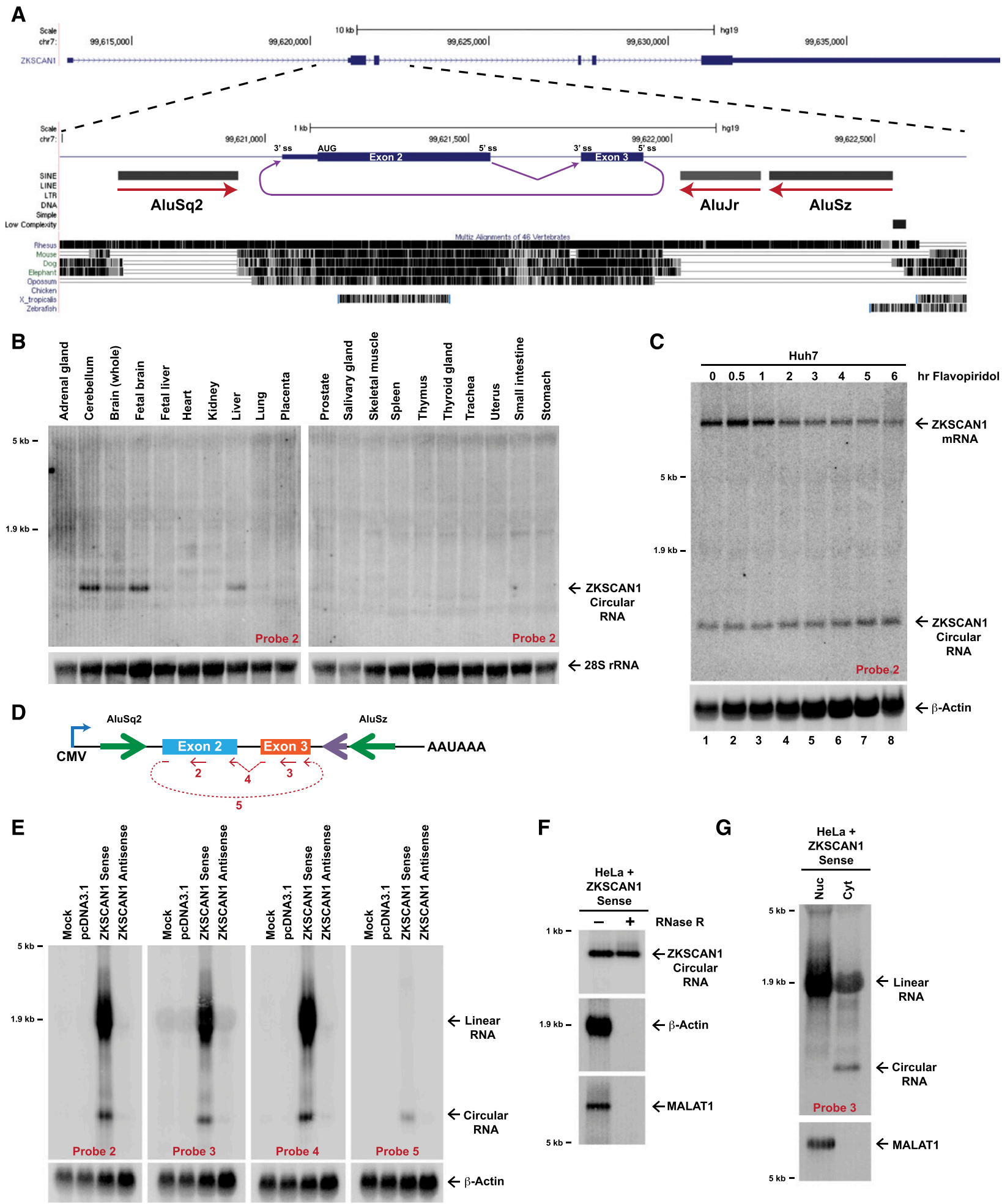

$\mathbf{F}$

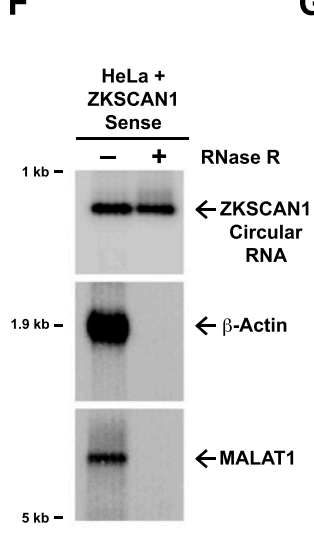

C
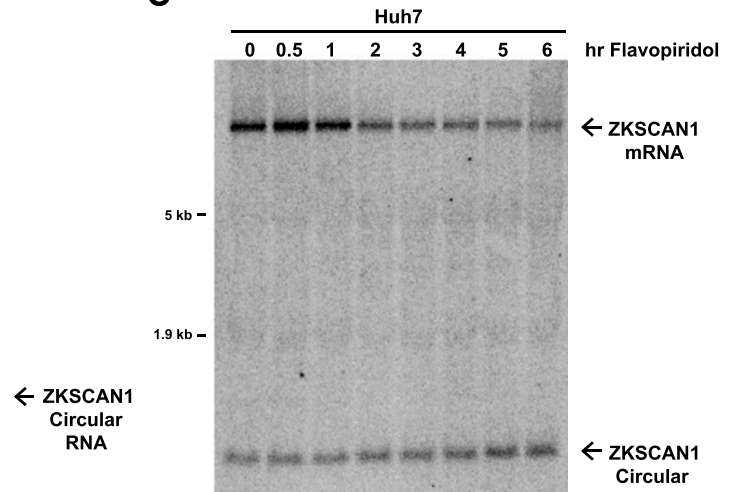

$\leftarrow 28$ S rRNA

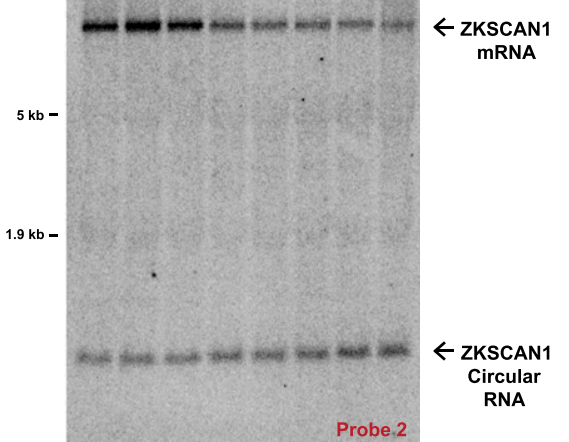
mRNA

Figure 1. The human ZKSCAN1 gene generates a circular RNA. (A) Exon/intron structure of the human ZKSCAN1 locus, highlighting a 2232-nt region that includes exons 2 and 3. A circular RNA is formed when the 5' splice site at the end of exon 3 is joined to the $3^{\prime}$ splice site at the beginning of exon 2 (purple). Repetitive elements in the designated orientations and evolutionary conservation patterns are shown. (B) Ten micrograms of total RNA from 20 normal human tissues was probed for ZKSCAN1 circular RNA expression. 28S ribosomal RNA was used as a loading control. $(C)$ Total RNA from Huh7 cells treated with flavopiridol, a transcriptional elongation inhibitor, was subjected to Northern blot analysis. $(D)$ The 2232-nt region of the ZKSCAN1 pre-mRNA was cloned into pcDNA3.1(+). The regions targeted by Northern oligonucleotide probes are denoted in red. (E) Plasmids containing the ZKSCAN1 region in the sense or antisense orientations were transfected into HeLa cells, and Northern blots were performed. $\beta$-Actin was used as a loading control. $(F)$ The ZKSCAN1 circular RNA is resistant to RNase R digestion. $(G)$ Transfected HeLa cells were fractionated to isolate nuclear and cytoplasmic total RNA, which was then subjected to Northern blot analysis with a probe to ZKSCAN1 exon 3. A probe to endogenous MALAT1 was used as a control for fractionation efficiency. 
RNA expression levels observed in these tissues are not simply by-products of increased transcription.

\section{Generation of an expression plasmid that accurately produces the ZKSCAN1 circular RNA}

Repeat sequences are commonplace in introns, and, indeed, there are 15 short interspersed elements (SINEs) in the intron upstream of ZKSCAN1 exon 2, and 13 downstream from exon 3 . Among these repeats, there are three primate-specific Alu elements in an inverted orientation that immediately flank the ZKSCAN1 circularizing exons (Fig. 1A). The upstream AluSq2 element is highly complementary to the downstream AluSz element (82\% identity over $287 \mathrm{nt}$ ) (Supplemental Fig. 1C) but not to the downstream AluJr element, which is a member of a separate subfamily of Alu elements. As these inverted Alu elements are located close to the exons that circularize, we hypothesized that relatively short introns may be sufficient to support circularization. A 2232-nt region of the ZKSCAN1 pre-mRNA, spanning from $547 \mathrm{nt}$ upstream of exon 2 to $796 \mathrm{nt}$ downstream from exon 3, was thus cloned into the pcDNA3.1 $(+)$ expression vector (Fig. 1D). It should be noted that this vector includes only a portion of the surrounding introns and does not include either of the surrounding exons (exons 1 or 4 ).

Expression vectors containing the 2232-nt insert in the sense or antisense orientation were transiently transfected into HeLa cells, and total RNA was isolated $24 \mathrm{~h}$ later. Circular RNA expression from the ZKSCAN1 sense plasmid was detected using Northern blots and four different oligonucleotide probes, including one (probe 5) complementary to the junction between the end of exon 3 and the beginning of exon 2 (Fig. 1D,E). Confirming that a true circular RNA was produced, the vector-derived transcript was resistant to digestion by the $3^{\prime}-5^{\prime}$ exonuclease RNase $\mathrm{R}$, unlike linear $\beta$-actin mRNA or the long noncoding RNA MALAT1 (Fig. 1F). In addition, RTPCR followed by Sanger sequencing confirmed that the 5' splice site of exon 3 was properly joined to the 3' splice site of exon 2 (Supplemental Fig. 1D). Finally, like the handful of other characterized circular RNAs (Hansen et al. 2013; Jeck et al. 2013; Memczak et al. 2013), the ZKSCAN1 circular RNA generated from our expression plasmid localized exclusively to the cytoplasm (Fig. 1G). We thus conclude that our plasmid generates bona fide ZKSCAN1 circular RNA. Circularization was additionally observed when the plasmid was transfected into mouse EpH4 cells (Supplemental Fig. 1E), indicating that the factors required to produce the ZKSCAN1 circular RNA are conserved between humans and mice.

\section{Short repeat sequences are sufficient for ZKSCAN1 circular RNA production}

Having shown that the intronic regions immediately surrounding exons 2 and 3 are sufficient for ZKSCAN1 circular RNA production, we next aimed to identify the minimal sequence elements. Focusing first on the upstream intron, we progressively deleted nucleotides from the 5' end, expecting to lose circular RNA production once a portion of the AluSq2 element (nucleotides 144439) was removed (Fig. 2A,B). Surprisingly, deleting the first $100 \mathrm{nt}$ of this intron resulted in a more than eightfold increase in ZKSCAN1 circular RNA levels and the production of almost no linear RNA, suggesting the presence of an element that strongly inhibits circularization (1002232 construct) (Fig. 2B). Cotransfection of a GFP expression plasmid confirmed equal transfection efficiency across samples (Supplemental Fig. 2A). Additional deletion constructs revealed that a cryptic $5^{\prime}$ splice site, located between nucleotides 14 and 23, was likely responsible for the inhibition (Supplemental Fig. 2B-D). Mutations that maintain the strength of the cryptic $5^{\prime}$ splice site had no effect, whereas single point mutations that disrupt the site were sufficient to completely relieve the repression on circularization (Supplemental Fig. 2E). This suggests that the intronic cryptic splice site may compete with the true splice sites for recognition by the spliceosome.

Upon continuing to delete from the $5^{\prime}$ end, we determined that the first $400 \mathrm{nt}$ were dispensable for ZKSCAN1 circular RNA production (Fig. 2B). Only 40 $\mathrm{nt}$ of the AluSq2 element (nucleotides 400-439) remain in the 400-2232 construct, indicating that a short repeat is able to support circularization as efficiently as the fulllength element (compare 400-2232 with 100-2232) (Fig. 2B). Circularization was only weakly observed upon deleting $410 \mathrm{nt}$ and was completely eliminated once $420 \mathrm{nt}$ or greater were removed (Fig. 2C). We therefore conclude that a small ( 30- 40-nt) region of the AluSq2 element is sufficient for ZKSCAN1 circular RNA production. Even higher circular RNA expression levels were obtained with constructs containing moderate-sized repeats (2002232, 250-2232, and 300-2232), although an additional transcript of $\sim 1.3 \mathrm{~kb}$ was observed (Fig. $2 \mathrm{~B}$ ). This RNA was resistant to digestion by RNase $\mathrm{R}$ and contains only exons 2 and 3, as determined by Northern blots and RTPCR (data not shown). This transcript may be a concatenated circular RNA corresponding to exons 2-3-2-3 that is generated by trans-splicing between two different primary transcripts or readthrough of the poly(A) signal (analogous to rolling circle replication). Alternatively, it may represent two independent 668-nt circles that are intertwined.

To then identify the minimal sequence elements present in the downstream intron, we generated an analogous set of constructs containing deletions from the 3' end (Fig. 2D; Supplemental Fig. 3B). Circularization was not observed when the complete AluSz element was deleted even if the AluJr element was present (400-1732 construct) (Fig. 2D). The AluJr element was, in fact, dispensable for circularization, whereas a 36-nt region of the AluSz element was sufficient to support ZKSCAN1 circular RNA production (400-1782 $\Delta 1479-1735$ construct) (Fig. 2D). Interestingly, the location of the minimal AluSz element in the downstream intron does not appear to be critical, as moving it $>250 \mathrm{nt}$ from its endogenous location (400-1782 construct) to immediately next to the $5^{\prime}$ splice site (400-1782 $\Delta 1479-1735$ construct) had no effect on circularization efficiency. Several additional deletion constructs (Supplemental Fig. 3C,D) allowed 
A

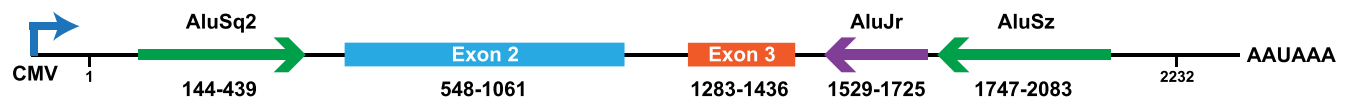

B

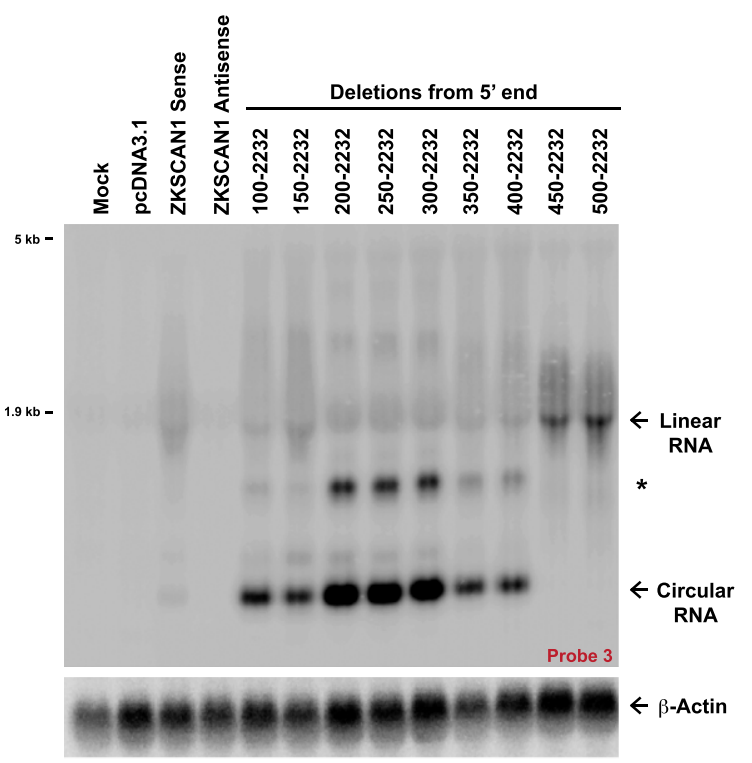

C

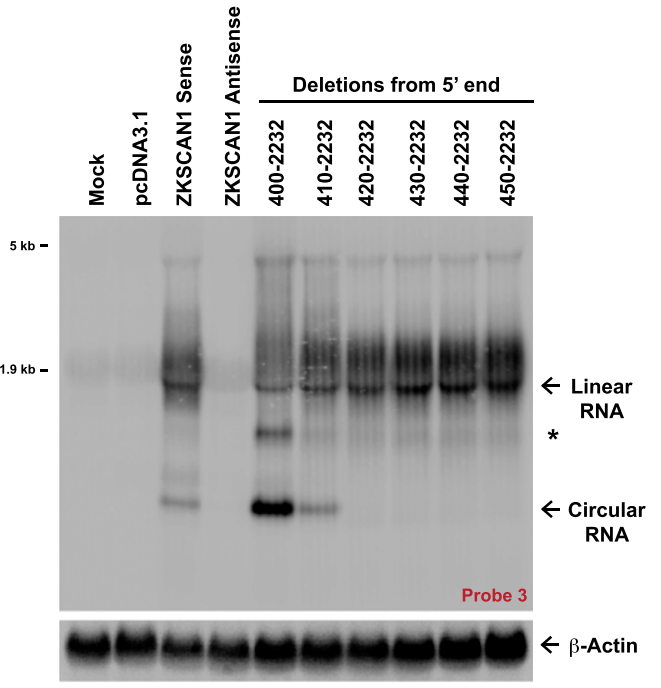

D

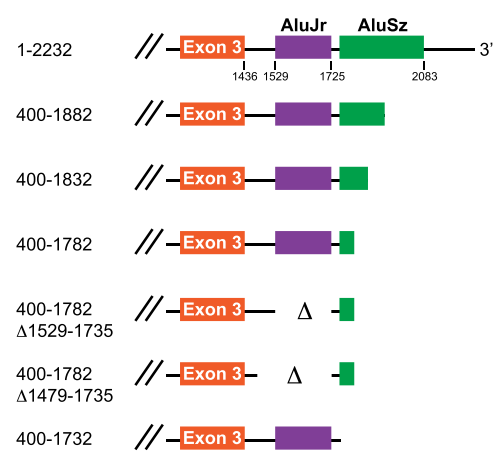

400-1832

400-1782

$400-1782$
$\Delta 1529-1735$

$400-1782$
$\Delta 1479-1735$

400-1732

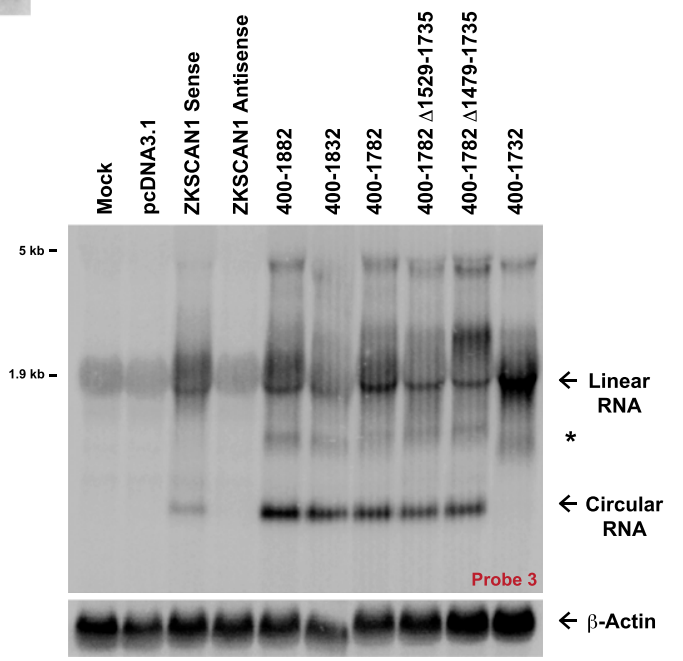

E

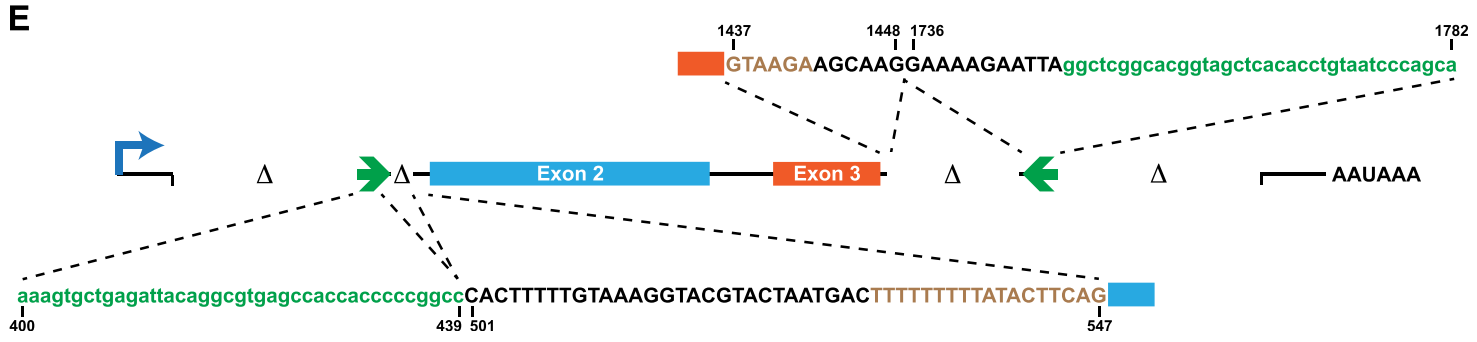

Figure 2. Short repeat sequences are sufficient to support ZKSCAN1 circularization. $(A)$ Numbering scheme for the ZKSCAN1 expression plasmid. $(B, C)$ ZKSCAN1 plasmids containing deletions at their $5^{\prime}$ ends were transfected into HeLa cells, and Northern blots were performed. The asterisk indicates an additional circular RNA species (see the text). (D) Using ZKSCAN1 expression plasmids starting at nucleotide 400, deletions were similarly introduced into the downstream intron. (E) Sequences of the minimal upstream and downstream introns that support circularization (400-1782 $\Delta 440-500 \Delta 1449-1735$ plasmid) are shown at the bottom and top, respectively. Repeat sequences (green) and splice sites (brown) are highlighted.

the identification of a final set of minimal introns that are sufficient to support efficient ZKSCAN1 circularization in cells (Fig. 2E). For the upstream intron (originally $547 \mathrm{nt}$ ),
$87 \mathrm{nt}$ are sufficient, which include $40 \mathrm{nt}$ of the AluSq2 element as well as the $3^{\prime}$ splice site and branch point sequences. For the downstream intron (originally $796 \mathrm{nt}$ ), 
$59 \mathrm{nt}$ are sufficient, which include the $5^{\prime}$ splice site and 36 nt of the AluSz element. Consistent with a role for the splicing machinery in ZKSCAN1 circularization, mutating the 5' splice site at the end of exon 3 eliminated ZKSCAN1 circular RNA production (Supplemental Fig. 3E). From these results, it is clear that $<40 \mathrm{nt}$ of intronic inverted repeats are sufficient to trigger spliceosome-mediated circularization at the ZKSCAN1 locus. Therefore, the repeat length requirements for ZKSCAN1 circularization are likely much simpler than those previously suggested to be required at the Sry locus (Dubin et al. 1995).

Base-pairing between the repeat elements is necessary for ZKSCAN1 circularization

As the minimal sufficient regions of the upstream AluSq2 and downstream AluSz elements are highly complementary, these sequences may base-pair and bring the splice sites into close proximity to one another (Fig. 3A). Consistent with this model, circularization was not observed when three of the base pairs between the repeats were disrupted (Mut 5' SINE and Mut 3' SINE) (Fig. 3A,B). When base-pairing was re-established by introduction of mutations in both repeats, a significant rescue in the level of the ZKSCAN1 circular RNA was detected (Mut 5' + 3' SINE) (Fig. 3A,B). This indicates that base-pairing between AluSq2 and AluSz is necessary for circularization. Although the repeats are highly complementary, there are seven mismatches over the minimal 36-nt region (Fig. 3A). Based on simple thermodynamics, we hypothesized that strengthening the hairpin between the repeats may increase the efficiency of circularization. However, two different perfectly complementary hairpins [Perfect (Mut 5') and Perfect (Mut 3')] (Fig. 3A) produced the circular RNA at levels similar to that obtained with the wild-type vector (Fig. 3C). This suggests that strengthening the hairpin over a certain thermodynamic threshold does not increase circularization efficiency. To test this threshold model further, the minimal repeats were converted to their reverse complement (Rev Comp) sequences (Fig. 3A). The Rev Comp hairpin is predicted to be more stable than the wild-type structure; surprisingly, almost no circular RNA expression was detected (Fig. 3C). We thus conclude that not every pair of inverted repeat elements is able to support circularization.

It is possible that a sequence-specific element critical for circularization may have been lost when the hairpin was converted to its Rev Comp. Arguing against this model, ZKSCAN1 circular RNA production was maintained when the perfect repeat sequences were scrambled (while maintaining base-pairing) (Supplemental Fig. 4). Furthermore, although nucleotides 400-439 of AluSq2 are sufficient for circularization, this minimal region is not required for circularization if other portions of the AluSq2/AluSz elements are present (Fig. 3D). The minimal sufficient repeats can, in fact, be replaced with unrelated 40-nt regions from the AluSq2/AluSz elements (Figs. 3E,F). However, analogous to above, not all 40-nt regions can support circularization (360-1826) (Fig. 3F), even when they are predicted to form hairpins stronger than other sequences that do support circularization.
Upon searching for features that distinguish between hairpins that do and do not support circularization, we noticed that both the Rev Comp (Fig. 3A) and 360-1826 (Fig. 3E) hairpins contain an increased number of G-U wobble base pairs. Replacing all of the wobble pairs in the Rev Comp hairpin with canonical Watson-Crick base pairs was sufficient to allow efficient circular RNA production (Supplemental Fig. 5, Rev Comp.5 construct). Interestingly, converting a single wobble pair to a canonical U-A base pair (Supplemental Fig. 5, Rev Comp.1 and Rev Comp.3) or even a U-C mismatch (Supplemental Fig. 5, Rev Comp.9/ was also sufficient, suggesting that subtle distortions in the hairpin specifically caused by G-U wobbles may significantly alter circularization efficiency. We thus conclude that more than simple thermodynamics is at play and that circularization is highly sensitive to how the repeat sequences base-pair to each other.

\section{Short repeats are sufficient for production of the HIPK3 circular RNA}

We next aimed to determine whether the rules for ZKSCAN1 circular RNA biogenesis can be applied more generally to explain how other circular RNAs are produced. The human HIPK3 gene has been implicated in multidrug resistance of cancer cells and encodes a serinethreonine kinase that regulates transcription and apoptosis (Begley et al. 1997; Curtin and Cotter 2004). Deep sequencing of several human cell lines previously suggested that HIPK3 also yields an abundant 1098-nt circular RNA comprised solely of exon 2 (Fig. 4A; Salzman et al. 2012; Jeck et al. 2013; Memczak et al. 2013). Using Northern blots and a probe complementary to the middle of exon 2, we determined that the HIPK3 circular RNA is most abundantly expressed in the brain, particularly in the cerebellum, but is also detectable in several other tissues (Fig. 4B). Like the ZKSCAN1 locus, highly complementary $\mathrm{Alu}$ repeats in an inverted orientation flank HIPK3 exon 2 (Fig. 4A; Supplemental Fig. 6A), suggesting that the biogenesis mechanisms for both RNAs may be similar.

We therefore cloned a 2803-nt region of the HIPK3 premRNA into pcDNA3.1(+) and confirmed that this expression vector efficiently generates a circular RNA when transfected into HeLa cells (Supplemental Fig. 6B,C). Consistent with the results from the ZKSCAN1 locus (Fig. 2), a 32-nt region of the upstream AluSz element (nucleotides 300-331) was sufficient for HIPK3 circular RNA production (Figs. 4D,E; Supplemental Fig. 6D). This region of AluSz is highly complementary to nucleotides 2470-2501 of the downstream AluSq2 element (Fig. 4C). Surprisingly, a HIPK3 plasmid ending at nucleotide 2503, thus containing this putative AluSq2-pairing site, failed to circularize (Supplemental Fig. 6E). In fact, deletions from the 3 ' end of the plasmid suggested that $>200-n t$ of the downstream AluSq2 element may be required for circularization (Supplemental Fig, 6E). However, this apparent discrepancy between minimal repeat lengths in the two introns was resolved upon the realization that the minimal AluSz region is also highly complementary 
A

WT
$\Delta \mathrm{G}=-46.30 \mathrm{kcal} / \mathrm{mol}$

Mut 5' SINE
$\Delta \mathrm{G}^{\prime}=-36.40 \mathrm{kcal} / \mathrm{mol}$

aaagugcugagauAUGaggegugagccaccacccceggec ||||||||||||||||||||||$|*|||$
acgacccuaauguccacacucgauggcacggcucgg

Mut 3' SINE
$\Delta \mathrm{G}=-37.10 \mathrm{kcal} / \mathrm{mol}$

Mut 5'+3' SINE

$\underset{\Delta G=-46.70 \mathrm{kcal} / \mathrm{mol}}{\text { Mut }}$

Perfect (Mut 5')
$\Delta G=-79.30 \mathrm{kcal} / \mathrm{mol}$

$\Delta G=-79.30 \mathrm{kcal} / \mathrm{mol}$

Perfect (Mut 3') $\Delta \mathrm{G}=-89.60 \mathrm{kcal} / \mathrm{mol}$

Rev Comp
$\Delta G=-59.30 \mathrm{kcal} / \mathrm{mo}$ |||||*||||||||*||||||*|||** || || uuucacgacucuaauguccgcacucgguggugggggecgg

D

$1-2232$

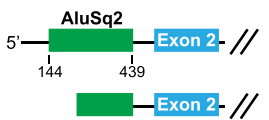

250-2232

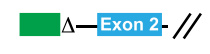

$250-2232$
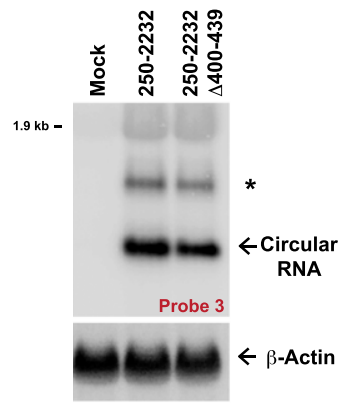

E

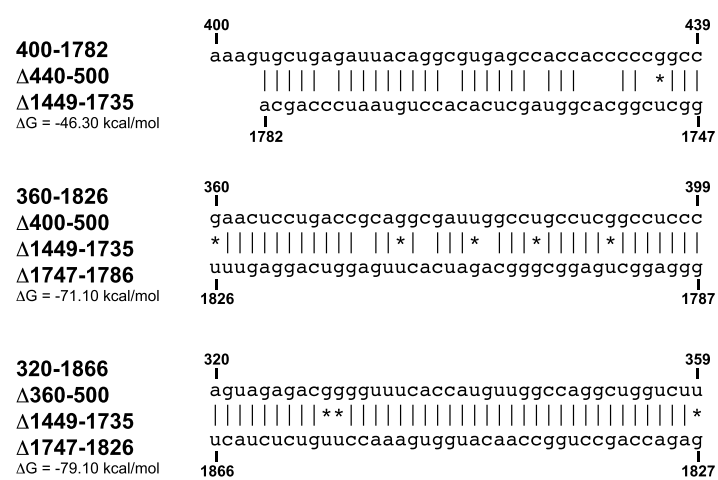

B
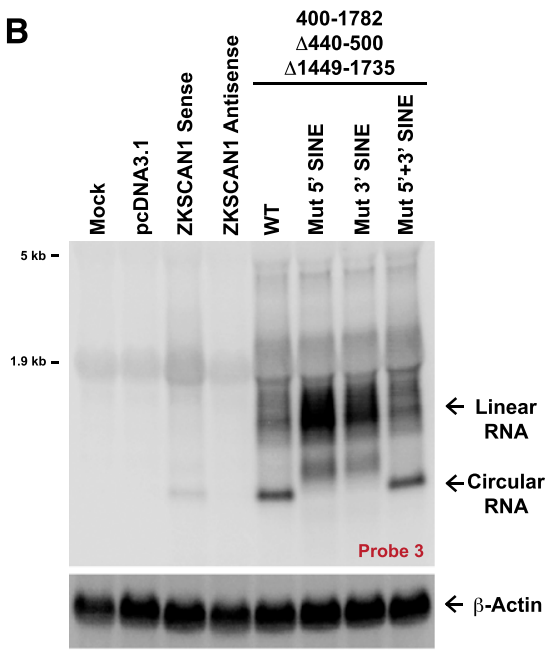

C

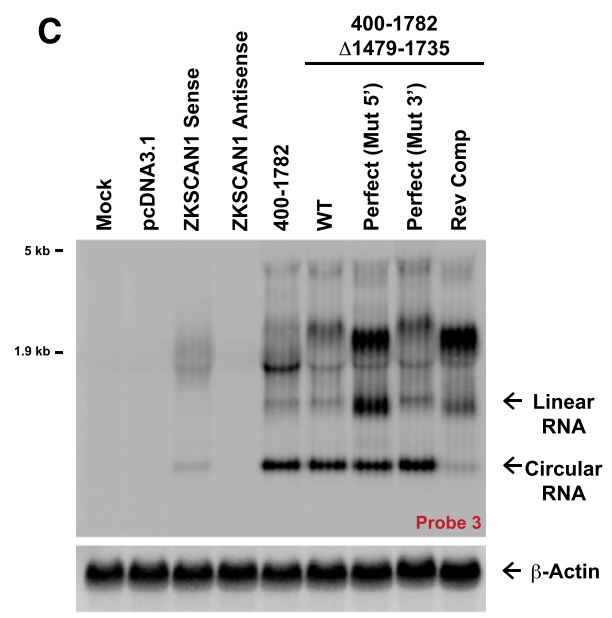

$\mathbf{F}$

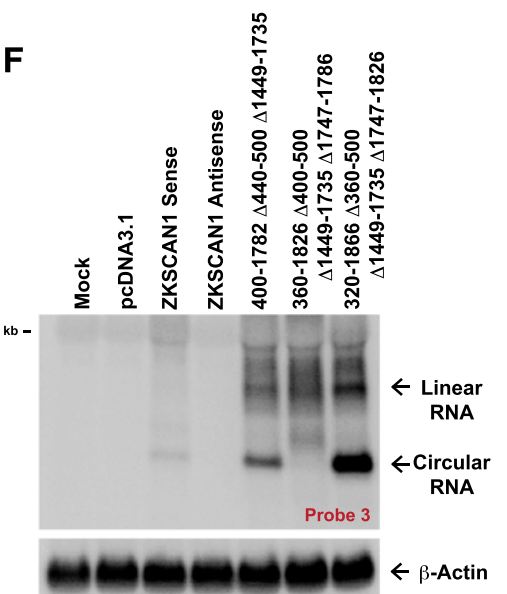

Figure 3. Only specific short repeat sequences are able to support circular RNA production. (A) Mutations (denoted in red) in the minimal sufficient repeat sequences were introduced into the ZKSCAN1 expression plasmid. mFold was used to calculate hairpin stabilities, assuming a 7-nt linker (AGAAUUA) between the two repeat sequences. $(B, C)$ ZKSCAN1 plasmids containing wild-type (WT) or mutant repeats were transfected into HeLa cells, and Northern blots were performed. The minimal sufficient introns (as depicted in Fig. 2E) were used in $B$, while slightly longer introns were used in $C$. $(D)$ Although sufficient for circular RNA production, the minimally sufficient AluSq2 region (nucleotides 400-439) was not required for circularization. (E) The minimal AluSq2/AluSz repeats (nucleotides 400-439 and 1747-1782) were replaced with other 40-nt regions of the Alu elements. The remainder of the plasmid was unchanged, allowing the effect of altering only the repeat sequences to be measured. mFold was used to calculate hairpin stabilities as above. $(F)$ Northern blots revealed that the thermodynamic stability of the hairpins is not an adequate predictor of circularization efficiency. 

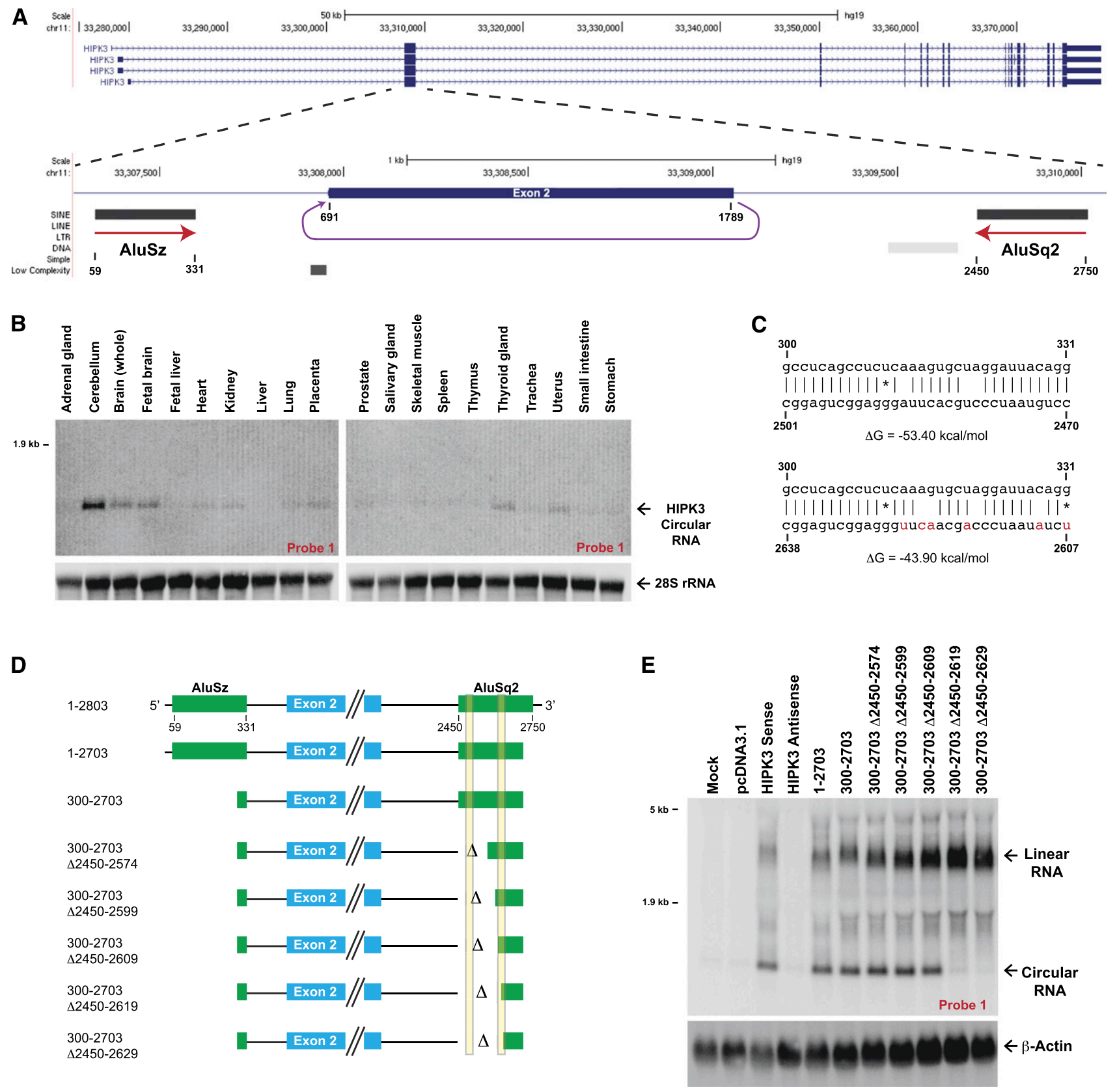

Figure 4. Specific short repeats are sufficient for HIPK3 circularization. $(A)$ Exon/intron structure of the human HIPK3 locus, highlighting a 2803-nt region that includes exon 2. A circular RNA is formed when the $5^{\prime}$ splice site at the end of exon 2 is joined to the 3 ' splice site at the beginning of exon 2 (purple). Repetitive elements in the designated orientations are shown. $(B)$ Ten micrograms of total RNA from 20 normal human tissues was probed for HIPK3 circular RNA expression. 28S ribosomal RNA was used as a loading control. (C) Nucleotides 300-331 of the upstream AluSz element are highly complementary to two different regions of the downstream AluSq2 element. Sequence differences between the two downstream elements are shown in red. (D) Deletions were introduced into the HIPK3 expression plasmid. The two AluSq2 complementary regions are highlighted in yellow. (E) HIPK3 plasmids were transfected into HeLa cells, and Northern blots were performed. Deleting portions of the 2607-2638 complementary region eliminated circular RNA production.

to nucleotides 2607-2638 of the downstream AluSq2 element (Fig. 4C). Whereas nucleotides 2470-2501 were dispensable for HIPK3 circularization, no circular RNA was produced when the 2607-2638 region was disrupted; e.g., by removing $13 \mathrm{nt}$ to generate the 300-2703 $\Delta 2450$ 2619 plasmid (Figs. 4D,E). We therefore conclude that base-pairing between nucleotides 300-331 of the upstream AluSz element and nucleotides 2607-2638 of the downstream AluSq2 element is sufficient to support HIPK3 circularization. Interestingly, the hairpin formed between the AluSz element and nucleotides 2607-2638 is predicted to be less stable than that formed with nucle- 
otides 2470-2501 (Fig. 4C), yet it is only the 2607-2638 region that supports circularization. In total, these results suggest that circularization at the HIPK3 and ZKSCAN1 loci have similarly short intronic repeat requirements.

\section{Sequences within longer repeats can inhibit} circularization

Our results from the ZKSCAN1 and HIPK3 loci clearly suggest that some, but not all, short repeats are able to support circular RNA production. As longer repeats contain more sequence and thus more potential for base-pairing, it seems likely that longer repeats might more uniformly support circularization. The EPHB4 locus, however, revealed that a longer repeat sequence can sometimes strongly inhibit circularization. EPHB4 encodes a receptor tyrosine kinase that is commonly overexpressed in human cancers (for review, see Noren and Pasquale 2007). In addition, a 360-nt circular RNA is generated from exons 11 and 12 (Jeck et al. 2013; Memczak et al. 2013; Salzman et al. 2013). Inverted Alu repeats flank these exons (Fig. 5A; Supplemental Fig. 7A), and we determined that a 1428-nt region of the EPHB4 pre-mRNA was sufficient for EPHB4 circular RNA production (Supplemental Fig. 7B-D). Initial deletions from the $5^{\prime}$ end of the EPHB4 expression plasmid suggested that $>100 \mathrm{nt}$ of the AluSx1 element may be required for circularization (Figs. 5B,C). However, analogous to the HIPK3 locus, only the middle of the AluSx1 element was critical for circularization, and a minimal sufficient region of <50 nt (nucleotides 200-250) was identified (200$1428 \Delta 251-343$ construct) (Fig. 5C).

Surprisingly, the 200-1428 EPHB4 expression plasmid, which contains $144 \mathrm{nt}$ of the AluSxl element, produced approximately fivefold less circular RNA than the 200$1428 \Delta 301-343$ plasmid, which contains only $100 \mathrm{nt}$ of the repeat (Fig. 5C). Similarly, the 250-1428 EPHB4 plasmid failed to generate the circular RNA but did upon deletion of nucleotides 301-343 (Fig. 5D). These results indicate that nucleotides $301-343$ of the repeat sequence can inhibit circularization. A poly(A) tract is present from nucleotide 321 to 343 , and deletion of this region was sufficient to allow the 250-1428 plasmid to produce the circular RNA (250-1428 $\Delta 321-343$ construct) (Fig. 5D). Binding of poly(A)-binding protein (PABP) to this region may sterically interfere with the ability of the hairpin to form. Alternatively, as a poly(A) tract is a low-complexity sequence, this portion of the repeat may bind more promiscuously to other transcripts in trans, thereby inhibiting circularization in cis.

Pre-mRNA 3' end formation is critical for circularization

As a circular RNA is covalently closed, the mature transcript contains no 5' cap or 3' poly(A) tail. Nevertheless, we found that disrupting the downstream polyadenylation signal in our expression plasmids (by placing it in the antisense orientation) caused the ZKSCAN1 circular RNA to no longer be produced (Fig. 6B). This suggested a potential role for $3^{\prime}$ end formation in circular
RNA biogenesis. ZKSCAN1 terminating in the noncanonical MALAT1 $3^{\prime}$ end processing mechanism, which involves recognition and cleavage of a tRNA-like structure by the endonuclease RNase P (Wilusz et al. 2008), was able to circularize (Fig. 6A,B). Therefore, circularization is dependent not on polyadenylation but instead on the act of forming a 3 ' end. After RNase P cleavage, the 3 ' end of MALAT1 is stabilized by a triple helical structure (Wilusz et al. 2012; Brown et al. 2014). We previously showed that when the triple helix is mutated, RNase P cleavage occurs, but the mature RNA is subsequently degraded (Wilusz et al. 2012), thereby uncoupling $3^{\prime}$ end cleavage from RNA stabilization. ZKSCAN1 pre-mRNA ending in the mutant triple helix produced a circular RNA, although at lower levels than the wild-type triple helix (Fig. 6B). We thus conclude that for a circular RNA to be produced from our expression plasmids, the pre-mRNA must be cleaved at its $3^{\prime}$ end. When the linear transcript has a stable $3^{\prime}$ end, greater levels of the circular RNA are produced. This suggests that circular RNA formation may at least in part occur post-transcriptionally.

\section{Circularization requires the intronic repeats to collaborate with the exons}

Having defined roles for intronic repeats and $3^{\prime}$ end formation signals in circular RNA biogenesis, we next addressed whether the exonic sequences play a role in circularization. A 51-nt artificial exon composed of restriction enzyme sites was inserted between the ZKSCAN1 minimal sufficient introns (as defined in Fig. 2E), thereby generating the "CircRNA Mini Vector" (Fig. 7A). No circular RNA was produced when this plasmid was transfected into HeLa cells (data not shown), indicating that additional exonic elements are required. Inserting most of exons 2 and 3 from ZKSCAN1 (nucleotides 548-1417) between the EcoRV and SacII sites restored efficient circular RNA production (Figs. 7A,B). As the internal intron between exons 2 and 3 (nucleotides 1062-1282) was not included in this vector, it is clear that the ZKSCAN1 minimal introns can support the production of circular RNAs comprised of a single or multiple exons. Inserting smaller ZKSCAN1 exonic regions into the CircRNA Mini Vector revealed that exon 3 (nucleotides 1283-1436) was dispensable for circularization (548-1047 construct) (Fig. 7B). Further decreasing the length of the inserted exon, however, eliminated circular RNA production. Whereas ZKSCAN1 exons $\geq 500 \mathrm{nt}$ in length circularized uniformly well, ZKSCAN1 exons $\leq 400 \mathrm{nt}$ circularized poorly or not at all (Fig. 7B; Supplemental Fig. 8). This suggests a correlation between exon length and circularization efficiency, a result that is consistent with previous computational analysis that indicated that longer than average exons are generally present in circular RNAs (Jeck et al. 2013).

We next determined whether the ZKSCAN1 minimal introns are able to support the circularization of exons derived from other genes. The ZKSCAN1 introns supported efficient production of the 360-nt EPHB4 exon 11/ 12 circular RNA (Fig. 7C), indicating that some smaller exons are able to circularize. Surrounding GAPDH exon 


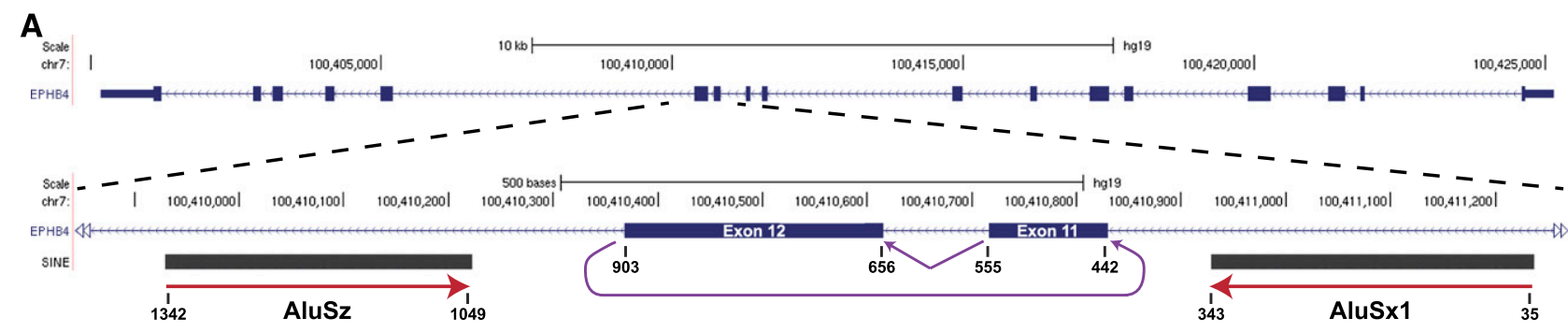

B

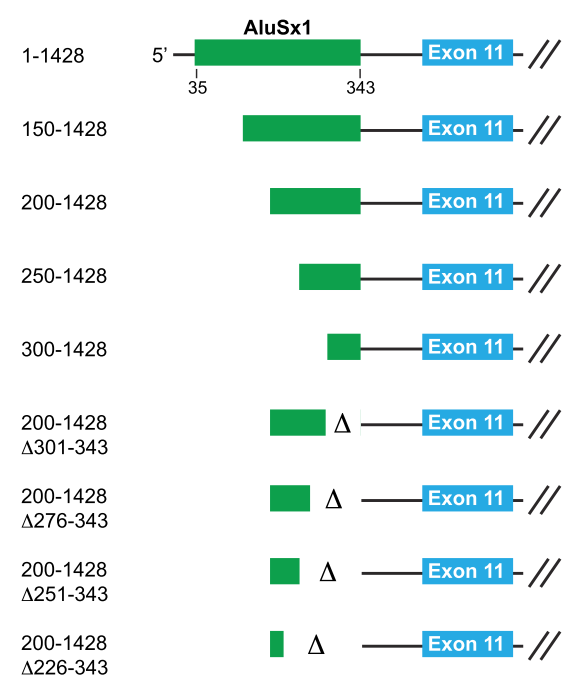

C

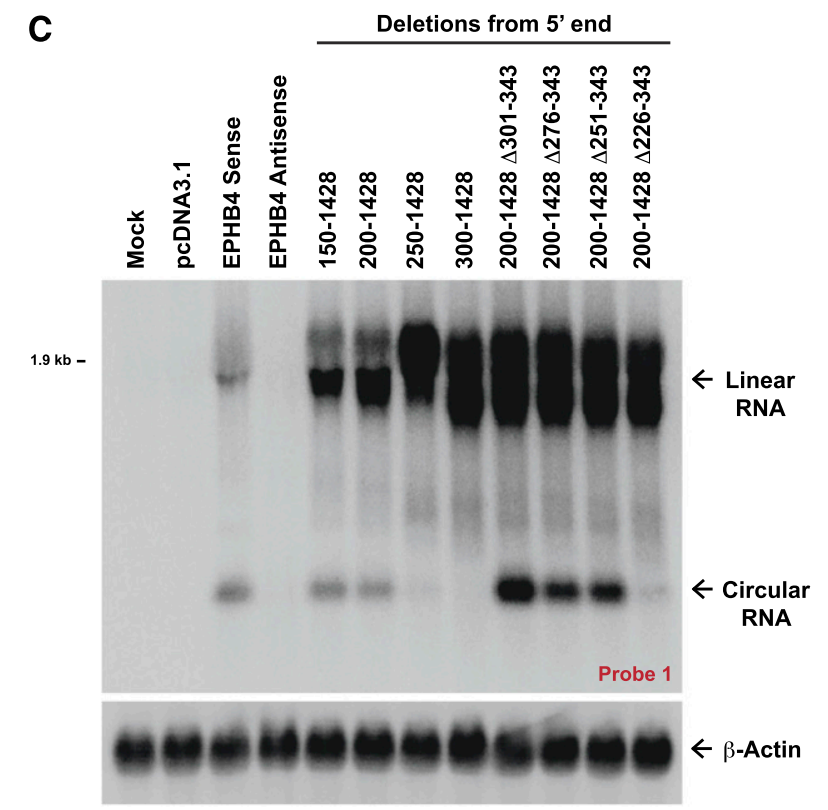

D

Deletions from 5' end

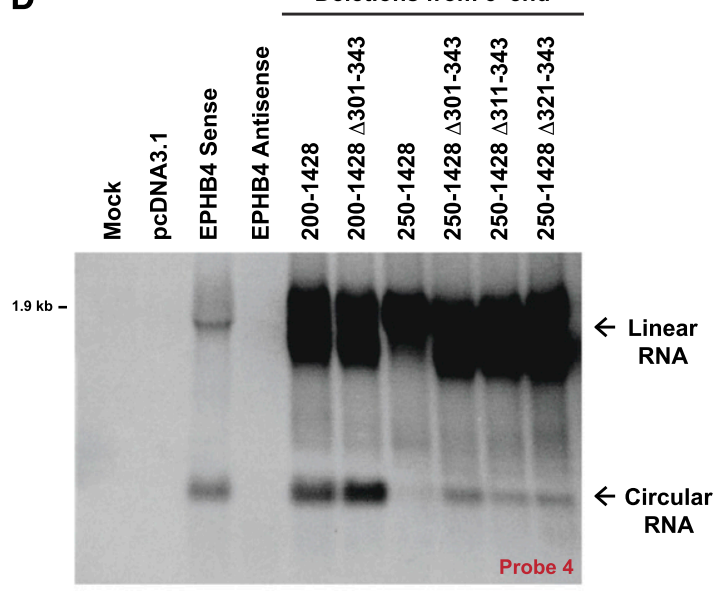

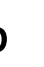

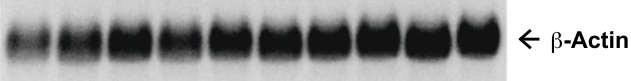

gagcgagactccatctcaggaaaaaaaaaaaaaaaaaaaaa

Figure 5. EPHB4 circularization is inhibited by a portion of the flanking Alu repeats. $(A)$ Exon/intron structure of the human EPHB4 locus, highlighting a 1428-nt region that includes exons 11 and 12. A circular RNA is formed when the $5^{\prime}$ splice site at the end of exon 12 is joined to the $3^{\prime}$ splice site at the beginning of exon 11 (purple). Repetitive elements in the designated orientations are shown. (B) Schematics of EPHB4 expression plasmids. Exon 12 and the downstream intron are not shown for simplicity. (C) EPHB4 plasmids were transfected into HeLa cells, and Northern blots were performed. Probe-binding sites are shown in Supplemental Figure 7C. The 2501428 plasmid contains $94 \mathrm{nt}$ of the AluSxl element, but no circularization was observed. In contrast, the 200-1428 $\Delta 251-343$ plasmid contains only $50 \mathrm{nt}$ of the AluSx1 element and efficiently generated the EPHB4 circular RNA. $(D)$ The poly(A) tract at the 3' end of the AluSx1 element (nucleotides 321-343) inhibits circularization. 
A
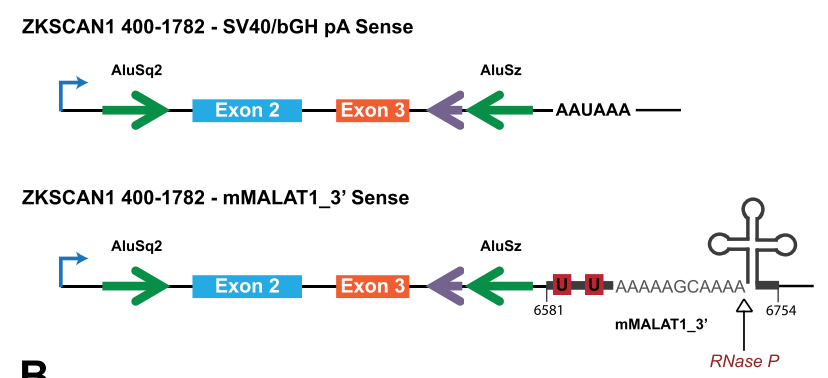

B

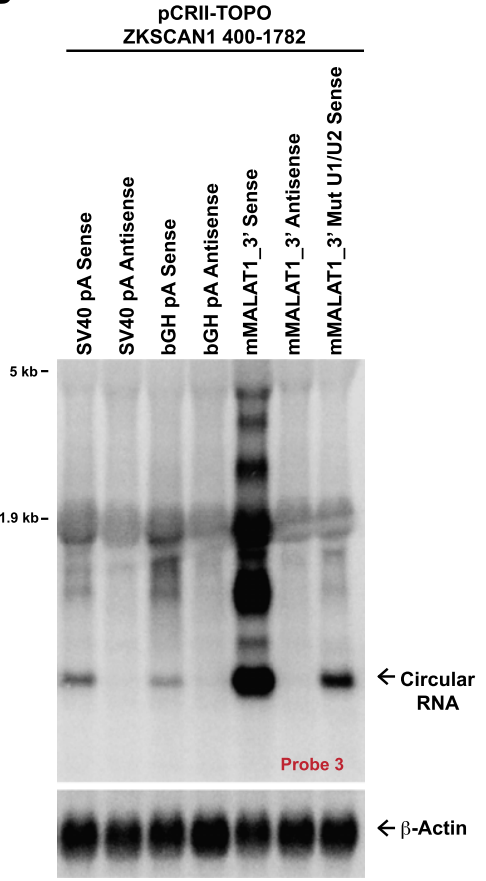

Figure 6. $3^{\prime}$ end formation is required for circularization. $(A)$ Schematics of ZKSCAN1 expression plasmids. The complete polyadenylation (pA) signals, which include the AAUAAA sequence, were placed in the antisense orientation or replaced by nucleotides 6581-6754 of mouse MALAT1. This 174-nt region, denoted as mMALAT1_3', includes a tRNA-like structure that is recognized and cleaved by RNase P (Wilusz et al. 2008). U-rich (denoted in red) and A-rich motifs then form a triple helical structure, thereby protecting the mature $3^{\prime}$ end from exonucleolytic degradation (Wilusz et al. 2012). To generate the mMALAT1_3' Mut U1/U2 plasmid, U $\rightarrow$ A mutations were introduced into both U-rich motifs, thereby disrupting the triple helix. (B) HeLa cells were transfected with pCRII-TOPO plasmids expressing ZKSCAN1 followed by differing 3'-terminal sequences. Northern blots were then performed using probe 3 from Figure 1D.

7, which does not naturally circularize, with the ZKSCAN1 minimal introns similarly resulted in circular RNA production (Fig. 7D). However, circularization was not observed when exon 2 of HIPK3, which is $1098 \mathrm{nt}$, was inserted (Fig. 7E). Short intronic repeats in their endogenous context were sufficient to support HIKP3 circularization (Fig. 4E), although the introns used in that plasmid were not as minimalized as those used here. From these various exonic sequences tested, we conclude that circularization clearly requires collaboration between the exons and their flanking introns.

\section{Discussion}

For many genes, pre-mRNA splicing can result in the production of a linear mRNA or a circular noncoding RNA (Fig. 7F). However, the mechanism by which the spliceosome selects only certain exons to circularize is largely unknown. In the present study, we demonstrated that short repeat elements in the flanking introns are critical for circularization of some intervening exons. The repeats must base-pair, which suggests that the splice sites must be brought into close proximity to one another to promote backsplicing. Although mechanistically simple, this step occurs in a highly selective manner, as the sequence of the repeats can drastically alter the efficiency of circular RNA production. Exons of longer length appear to circularize more efficiently, and 3' end processing is required for circularization, suggesting that circular RNA production may occur post-transcriptionally. Our data thus provide new insights into how to produce a circular RNA as well as how the critical choice between linear versus circular RNA is regulated.

\section{A proposed general model for how circular RNAs are produced}

Although recent deep sequencing studies have clearly revealed that many genes produce circular RNAs, our current understanding of the circularization mechanism is rather limited and based largely on a single gene: mouse Sry. The Sry exon is flanked by consensus splice sites as well as $>15-\mathrm{kb}$ inverted repeats (Capel et al. 1993). This rather unusual genomic organization led Capel et al. (1993) to propose that the repeats form an intramolecular stem-loop structure, resulting in the positioning of the splice donor close to the splice acceptor. Consistent with this model, complete deletion of either of the inverted repeats eliminated Sry exon circularization in cells (Dubin et al. 1995). Although 400 complementary nucleotides were proposed to be necessary to produce the Sry circular RNA (Dubin et al. 1995), smaller repeat sequences were not tested. Based on these results from the Sry locus, a ciRS-7 (also known as CDR1as) circular RNA expression plasmid was recently generated by artificially flanking the exon with $\sim 800 \mathrm{nt}$ of perfectly complementary repeats (Hansen et al. 2013). However, such long repeats do not naturally flank the ciRS-7 exon or the large majority of other exons. Smaller inverted repeats, particularly Alu elements that are $\sim 300 \mathrm{nt}$ in length, are statistically enriched in introns flanking exons that circularize (Jeck et al. 2013), although these repeats have yet to be experimentally shown to facilitate the production of circular RNAs.

Based on our results from the ZKSCAN1, HIPK3, and EPHB4 genes, we built on these prior studies to propose the following model for how the spliceosome determines whether to produce a linear mRNA or a circular noncoding RNA (Fig. 7F). Although the most common outcome of 
$\mathbf{A}$

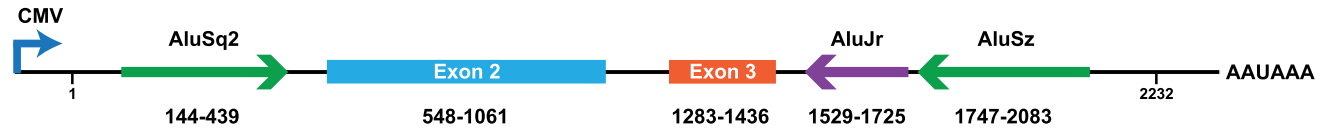

400-1782 $\Delta 440-500 \Delta 1449-1735$ Vector:
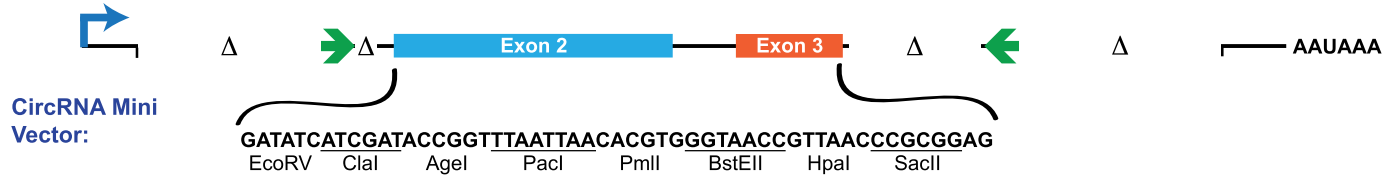

B

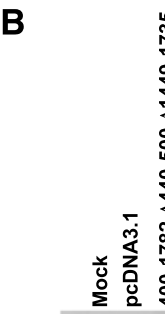

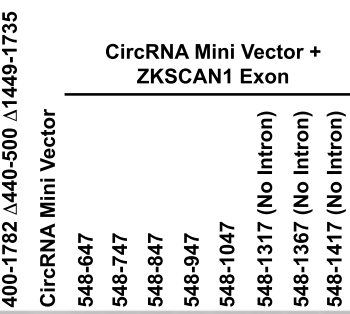
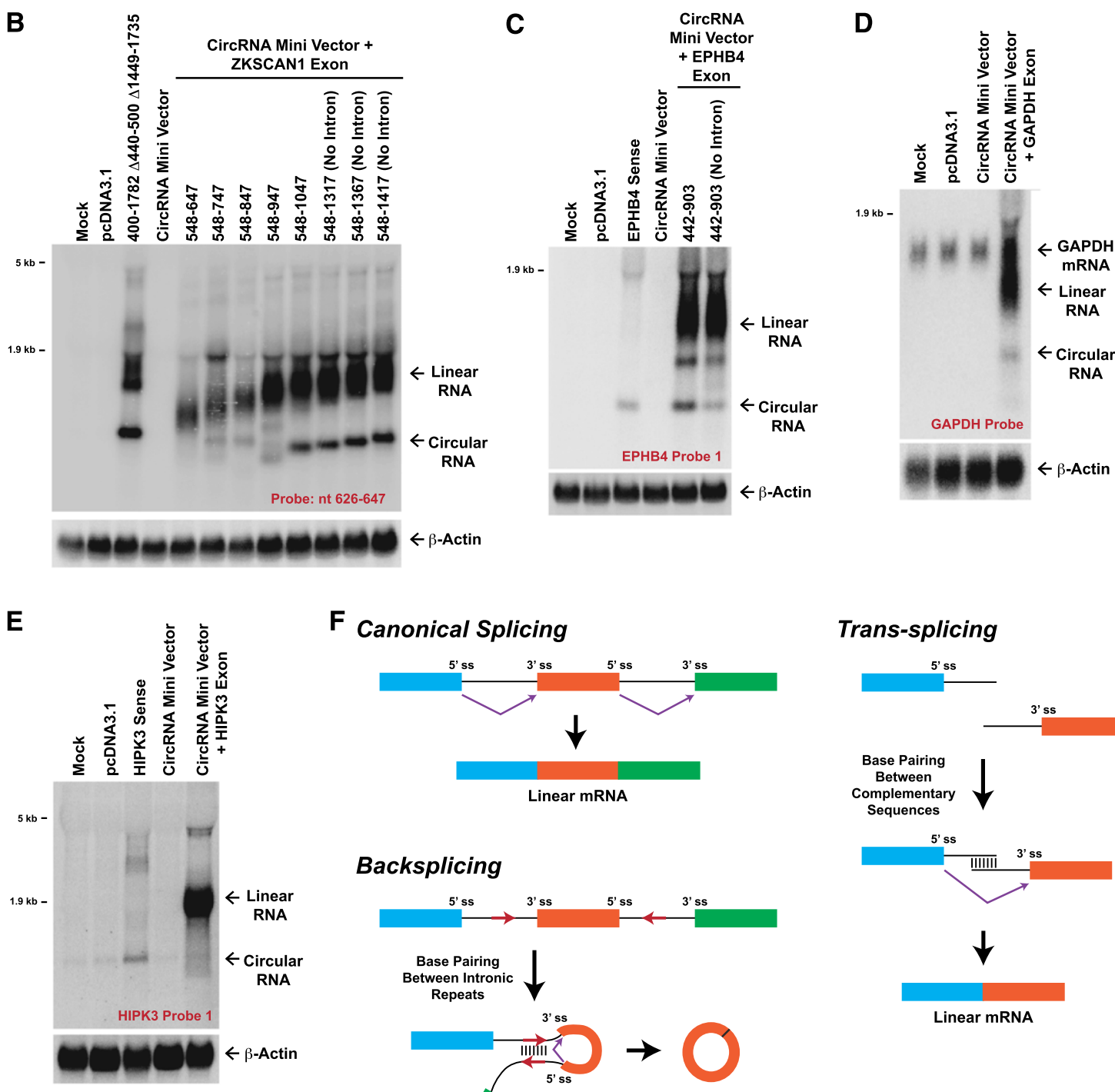

F Canonical Splicing

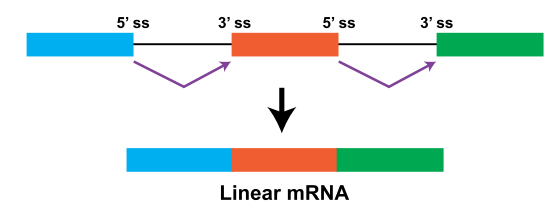

Backsplicing

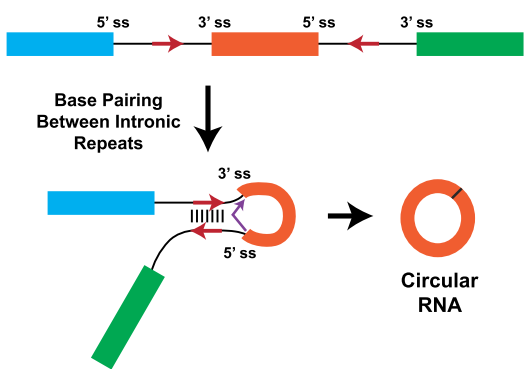

Trans-splicing

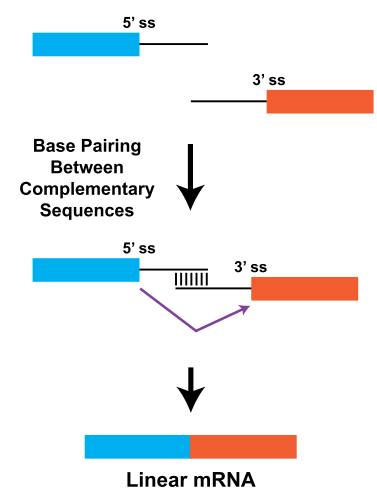

Figure 7. Collaboration between the exon and introns allows circularization. $(A)$ To facilitate the identification of exon sequences that can be circularized, exons 2 and 3 of the minimal ZKSCAN1 400-1782 $\Delta 440-500 \Delta 1449-1735$ expression plasmid were replaced with an artificial 51-nt exon composed of restriction enzyme sites. (B) Segments of the ZKSCAN1 exons were then inserted between the EcoRV and SacII sites. The numbering scheme is as in $A$ and Figure 2A. The intron between exons 2 and 3 (nucleotides 1062-1282) was not included in the vectors designated "No Intron." HeLa cells were then transfected, and Northern blots were performed. (C) Exons 11 and 12 of EPHB4 with or without the internal intron were inserted into the CircRNA Mini Vector. The numbering scheme is as per Figure 5A. (D) Exon 7 of GAPDH, which is $336 \mathrm{nt}$, was inserted into the CircRNA Mini Vector. In addition to the circular RNA, the endogenous GAPDH transcript was detected on the Northern blot. $(E)$ The minimal ZKSCAN1 introns were unable to support circularization of HIKP3 exon 2. $(F)$ Comparison of different pre-mRNA splicing mechanisms. Whereas canonical splicing produces a linear mRNA, base-pairing between intronic repeat sequences can trigger backsplicing and the formation of a circular RNA. When base-pairing occurs between complementary sequences on two independent primary transcripts, trans-splicing can generate a chimeric linear mRNA. 
splicing appears to be linear mRNA production, circularization is initiated when repeats in the introns immediately flanking the exons base-pair to one another (Fig. 3). As was proposed at the Sry locus, this brings the intervening splice sites into close proximity, facilitating catalysis. Short inverted repeats $(\sim 30-40 \mathrm{nt})$ are sufficient, although more than simple thermodynamics regulates this critical event. First, the sequence content of the repeats can significantly alter circularization efficiency (Figs. 3-5). Low-complexity sequences [such as poly(A) tracts] or subtle distortions in the hairpin between the repeats (e.g., due to G-U wobble pairs) can inhibit circularization. Additional RNA secondary structures and RNA-binding proteins likely also play significant roles in determining whether a given repeat is accessible. Second, given that $3^{\prime}$ end processing was required for circularization from our plasmids (Fig. 6), the process probably occurs post-transcriptionally. Backsplicing thus appears to be a relatively slow process, and the flanking introns likely must be spliced slower than average. If there is not sufficient time for the repeats to base-pair and/or recruit the spliceosome, a linear mRNA is produced. Third, the intronic repeats must collaborate with the exonic sequences (Fig. 7). In general, longer exons appear to circularize more efficiently than shorter exons, possibly because their increased length provides flexibility to the transcript and allows the intronic repeats to base-pair more easily. Due to these multiple regulatory steps, circularization is thus restricted to only specific exons despite the fact that repeat sequences comprise $>45 \%$ of the human genome and are commonplace in introns.

Repeats play a critical role in shaping exon circularization patterns

Although Alu elements flank the ZKSCAN1, HIPK3, and EPHB4 exons that circularize, other classes of repeat sequences are likely able to support circularization. For example, we found that inverted DNA transposon repeats are sufficient to support circularization at the human GCN1L1 locus (Supplemental Fig. 9). Furthermore, considering that the ZKSCAN1 circular RNA was still produced after scrambling the flanking repeat sequences (Supplemental Fig. 4), it is highly likely that complementary sequences that are not annotated repeats may be able to support circularization. However, it should be noted that the simple presence of a pair of inverted repeats does not mean that a circular RNA will be produced at a similar efficiency in all cell types. For example, we observed that the ratio of the ZKSCAN1 mRNA versus the circular RNA present in various tissues varied significantly (Supplemental Fig. 1B). This may be because the transcripts have different half-lives across cell types or because intron pairing between the repeats efficiently occurs only under certain conditions. RNA-binding proteins, especially those that bind dsRNA, may modulate the accessibility of the repeat sequences to base-pair. For example, ADAR (adenosine deaminase acting on RNA) enzymes may generally inhibit circular RNA production, as they convert adenosines in double-stranded regions to inosines, thereby unwinding hairpins (for review, see Nishikura 2010). Supporting this model, A-to-I editing is most commonly observed in intronic repeats that are engaged in intramolecular base-pairing (Athanasiadis et al. 2004), exactly the sort of structure that is needed for circularization. Further studies are needed to understand how base-pairing between the introns may affect spliceosome assembly as well as the recruitment of wellcharacterized splicing regulatory factors, including hnRNP and SR proteins.

\section{Interplay between $3^{\prime}$ end formation and circularization}

In addition to the intronic inverted repeat sequences, a functional $3^{\prime}$ end processing signal was surprisingly found to be required for circularization from our expression plasmids, suggesting that circularization may occur post-transcriptionally (Fig. 6). Although most introns are thought to be spliced cotranscriptionally (for review, see Brugiolo et al. 2013), there is increasing evidence that some introns are not spliced until after polyadenylation occurs (Vargas et al. 2011; Bhatt et al. 2012). These incompletely spliced, polyadenylated transcripts accumulate in the chromatin faction and, after a significant lag period, are eventually spliced to produce functional mRNAs. Although some of these post-transcriptional splicing events are due to functionally impaired splicing signals, there is evidence that alternatively spliced exons can also be slowly spliced (Vargas et al. 2011). This suggests that regulatory events, such as the binding of alternative splicing regulatory proteins, can cause transcription and splicing to become uncoupled. We propose that the kinetics of splicing are likely slowed during the circularization process, possibly because pairing of the intronic repeats interferes with exon definition, thereby preventing the cotranscriptional formation of a fully spliced linear mRNA. By forming a stable $3^{\prime}$ end, the transcript is able to avoid degradation by nuclear RNA surveillance mechanisms (for review, see Schmidt and Butler 2013) and allow the spliceosome and other regulatory factors to determine whether to produce a linear mRNA or a circular RNA. Alternatively, as polyadenylation and splicing can be functionally coupled (Niwa and Berget 1991), the $3^{\prime}$ end processing machinery may recruit or otherwise directly interact with circular RNA biogenesis factors, thereby promoting circularization.

In summary, our findings provide key insights into the mechanism by which the spliceosome generates circular RNAs. Considering that thousands of circular RNAs are produced in eukaryotic cells, base-pairing between intronic repeats appears to be a widespread phenomenon by which the transcriptome is expanded. Interestingly, although the exonic sequences of protein-coding genes may be highly evolutionarily conserved, the genomic repeat landscape varies significantly among species (for review, see Shedlock and Okada 2000). Therefore, the production of a circular RNA from a given exon can be highly species-specific, and very different functional 
RNAs, some of which are circular, may be produced from a given gene. Exon circularization thus likely represents an underappreciated way by which the coding capacity of eukaryotic genes is expanded and the ultimate functions of genes are modulated.

\section{Materials and methods}

\section{Expression plasmid construction}

To generate the ZKSCAN1, HIPK3, and EPHB4 expression plasmids, the indicated sequences were inserted into pcDNA3.1(+) (Life Technologies) as further described in the Supplemental Material. Genomic coordinates in human genome version 19 (hg19) for the full-length inserts were as follows: ZKSCAN1 (chr7: 99,620,495-99,622,726), HIPK3 (chr11: 33,307,26933,310,071), and EPHB4 (chr7: 100,409,844-100,411,271). For the CircRNA Mini Vector depicted in Figure 7A, exonic sequences were inserted between the minimal ZKSCAN1 introns using the EcoRV and SacII sites. To generate ZKSCAN1 plasmids terminating in various $3^{\prime}$ end sequences (bGH polyadenylation signal, SV40 polyadenylation signal, or the mMALAT1_3' region), the cGFP ORF in our previously described pCRII-TOPO CMV-cGFP expression plasmids (Wilusz et al. 2012) was replaced with the designated region of ZKSCAN1. Additional details and the sequences of the inserts for all plasmids are provided in the Supplemental Material. mFold was used to calculate hairpin stabilities, assuming a 7-nt linker (AGAAUUA) between the two repeat sequences.

\section{Transfections and RNA analysis}

HeLa and Huh7 cells were grown at $37^{\circ} \mathrm{C}$ and $5 \% \mathrm{CO}_{2}$ in Dulbecco's modified Eagle's medium (DMEM) containing high glucose (Life Technologies), supplemented with penicillin-streptomycin and $10 \%$ fetal bovine serum. One microgram of each expression plasmid was transfected using Lipofectamine 2000 (Life Technologies), and total RNA was isolated using Trizol (Life Technologies) as per the manufacturer's instructions. Nuclear and cytoplasmic fractionation was performed as previously described (Wilusz et al. 2008). To inhibit RNA polymerase II transcription, Huh7 cells were treated with flavopiridol $(1 \mu \mathrm{M}$ final concentration; Sigma) for $0-6 \mathrm{~h}$ at $37^{\circ} \mathrm{C}$. For RNase R treatments, $10 \mu \mathrm{g}$ of total RNA was treated with $20 \mathrm{U}$ of RNase R (Epicentre) for $1 \mathrm{~h}$ at $37^{\circ} \mathrm{C}$. Northern blots using oligonucleotide probes were performed as previously described (Wilusz et al. 2008). All oligonucleotide probe sequences are provided in Supplemental Table 1.

The Human Total RNA Master Panel II (Clontech) was used to examine the expression of endogenous circular RNAs in normal human tissues. To measure ZKSCAN1 circular RNA and mRNA levels, reverse transcription using random hexamers and qPCR was performed as previously described (Sunwoo et al. 2009). qPCR primer sequences are provided in Supplemental Table 1.

\section{Acknowledgments}

We thank Sara Cherry, Kristen Lynch, Phillip Sharp, Deirdre Tatomer, and Jeff Wilusz for discussions and advice. This work was supported by National Institutes of Health (NIH) grant R00GM104166 (to J.E.W.) and start-up funds from the University of Pennsylvania. Early stages of this research was performed at Massachusetts Institute of Technology by J.E.W. and was supported in part by NIH grants R01-GM34277 and R01-CA133404 to Phillip Sharp, J.E.W.'s post-doctoral advisor.

\section{References}

Athanasiadis A, Rich A, Maas S. 2004. Widespread A-to-I RNA editing of Alu-containing mRNAs in the human transcriptome. PLoS Biol 2: e391.

Begley DA, Berkenpas MB, Sampson KE, Abraham I. 1997. Identification and sequence of human PKY, a putative kinase with increased expression in multidrug-resistant cells, with homology to yeast protein kinase Yak1. Gene 200: 35-43.

Bhatt DM, Pandya-Jones A, Tong AJ, Barozzi I, Lissner MM, Natoli G, Black DL, Smale ST. 2012. Transcript dynamics of proinflammatory genes revealed by sequence analysis of subcellular RNA fractions. Cell 150: 279-290.

Braun S, Domdey H, Wiebauer K. 1996. Inverse splicing of a discontinuous pre-mRNA intron generates a circular exon in a HeLa cell nuclear extract. Nucleic Acids Res 24: 4152-4157.

Brown JA, Bulkley D, Wang J, Valenstein ML, Yario TA, Steitz TA, Steitz JA. 2014. Structural insights into the stabilization of MALAT1 noncoding RNA by a bipartite triple helix. Nat Struct Mol Biol 21: 633-640.

Brugiolo M, Herzel L, Neugebauer KM. 2013. Counting on cotranscriptional splicing. F1000Prime Rep 5: 9.

Burd CE, Jeck WR, Liu Y, Sanoff HK, Wang Z, Sharpless NE. 2010. Expression of linear and novel circular forms of an INK4/ARF-associated non-coding RNA correlates with atherosclerosis risk. PLoS Genet 6: e1001233.

Capel B, Swain A, Nicolis S, Hacker A, Walter M, Koopman P, Goodfellow P, Lovell-Badge R. 1993. Circular transcripts of the testis-determining gene Sry in adult mouse testis. Cell 73: 1019-1030.

Cech TR, Steitz JA. 2014. The noncoding RNA revolutiontrashing old rules to forge new ones. Cell 157: 77-94.

Chen CY, Sarnow P. 1995. Initiation of protein synthesis by the eukaryotic translational apparatus on circular RNAs. Science 268: 415-417.

Cocquerelle C, Mascrez B, Hetuin D, Bailleul B. 1993. Missplicing yields circular RNA molecules. FASEB I 7: 155-160.

Curtin JF, Cotter TG. 2004. JNK regulates HIPK3 expression and promotes resistance to Fas-mediated apoptosis in DU 145 prostate carcinoma cells. J Biol Chem 279: 17090-17100.

Danan M, Schwartz S, Edelheit S, Sorek R. 2012. Transcriptomewide discovery of circular RNAs in Archaea. Nucleic Acids Res 40: 3131-3142.

Dubin RA, Kazmi MA, Ostrer H. 1995. Inverted repeats are necessary for circularization of the mouse testis Sry transcript. Gene 167: 245-248.

Guo JU, Agarwal V, Guo H, Bartel DP. 2014. Expanded identification and characterization of mammalian circular RNAs. Genome Biol 15: 409.

Hansen TB, Jensen TI, Clausen BH, Bramsen JB, Finsen B, Damgaard CK, Kjems J. 2013. Natural RNA circles function as efficient microRNA sponges. Nature 495: 384-388.

Jeck WR, Sharpless NE. 2014. Detecting and characterizing circular RNAs. Nat Biotechnol 32: 453-461.

Jeck WR, Sorrentino JA, Wang K, Slevin MK, Burd CE, Liu J, Marzluff WF, Sharpless NE. 2013. Circular RNAs are abundant, conserved, and associated with ALU repeats. RNA 19: 141-157.

Memczak S, Jens M, Elefsinioti A, Torti F, Krueger J, Rybak A, Maier L, Mackowiak SD, Gregersen LH, Munschauer M, et al. 2013. Circular RNAs are a large class of animal RNAs with regulatory potency. Nature 495: 333-338.

Mulligan MK, Wang X, Adler AL, Mozhui K, Lu L, Williams RW. 2012. Complex control of GABA(A) receptor subunit mRNA expression: variation, covariation, and genetic regulation. PLOS ONE 7: e34586. 
Nigro JM, Cho KR, Fearon ER, Kern SE, Ruppert JM, Oliner JD, Kinzler KW, Vogelstein B. 1991. Scrambled exons. Cell 64: 607-613.

Nishikura K. 2010. Functions and regulation of RNA editing by ADAR deaminases. Annu Rev Biochem 79: 321-349.

Niwa M, Berget SM. 1991. Mutation of the AAUAAA polyadenylation signal depresses in vitro splicing of proximal but not distal introns. Genes Dev 5: 2086-2095.

Noren NK, Pasquale EB. 2007. Paradoxes of the EphB4 receptor in cancer. Cancer Res 67: 3994-3997.

Pasman Z, Been MD, Garcia-Blanco MA. 1996. Exon circularization in mammalian nuclear extracts. RNA 2: 603-610.

Salzman J, Gawad C, Wang PL, Lacayo N, Brown PO. 2012. Circular RNAs are the predominant transcript isoform from hundreds of human genes in diverse cell types. PLOS ONE 7: e30733.

Salzman J, Chen RE, Olsen MN, Wang PL, Brown PO. 2013. Cell-type specific features of circular RNA expression. PLOS Genet 9: e1003777.

Schindewolf C, Braun S, Domdey H. 1996. In vitro generation of a circular exon from a linear pre-mRNA transcript. Nucleic Acids Res 24: 1260-1266.

Schmidt K, Butler JS. 2013. Nuclear RNA surveillance: role of TRAMP in controlling exosome specificity. Wiley Interdiscip Rev RNA 4: 217-231.

Shedlock AM, Okada N. 2000. SINE insertions: powerful tools for molecular systematics. BioEssays 22: 148-160.

Sunwoo H, Dinger ME, Wilusz JE, Amaral PP, Mattick JS, Spector DL. 2009. MEN $\varepsilon / \beta$ nuclear-retained non-coding RNAs are up-regulated upon muscle differentiation and are essential components of paraspeckles. Genome Res 19: 347-359.

van Dekken H, Tilanus HW, Hop WC, Dinjens WN, Wink JC, Vissers KJ, van Marion R. 2009. Array comparative genomic hybridization, expression array, and protein analysis of critical regions on chromosome arms $1 \mathrm{q}, 7 \mathrm{q}$, and $8 \mathrm{p}$ in adenocarcinomas of the gastroesophageal junction. Cancer Genet Cytogenet 189: 37-42.

Vargas DY, Shah K, Batish M, Levandoski M, Sinha S, Marras SA, Schedl P, Tyagi S. 2011. Single-molecule imaging of transcriptionally coupled and uncoupled splicing. Cell 147: 1054-1065.

Wang PL, Bao Y, Yee MC, Barrett SP, Hogan GJ, Olsen MN, Dinneny JR, Brown PO, Salzman J. 2014. Circular RNA is expressed across the eukaryotic tree of life. PLOS ONE 9: e90859.

Wilusz JE, Sharp PA. 2013. Molecular biology. A circuitous route to noncoding RNA. Science 340: 440-441.

Wilusz JE, Freier SM, Spector DL. 2008. 3' end processing of a long nuclear-retained noncoding RNA yields a tRNA-like cytoplasmic RNA. Cell 135: 919-932.

Wilusz JE, Sunwoo H, Spector DL. 2009. Long noncoding RNAs: functional surprises from the RNA world. Genes Dev 23: 1494-1504.

Wilusz JE, Jnbaptiste CK, Lu LY, Kuhn CD, Joshua-Tor L, Sharp PA. 2012. A triple helix stabilizes the $3^{\prime}$ ends of long noncoding RNAs that lack poly(A) tails. Genes Dev 26: 2392-2407.

Yang L, Froberg JE, Lee JT. 2014. Long noncoding RNAs: fresh perspectives into the RNA world. Trends Biochem Sci 39: 35-43.

Zaphiropoulos PG. 1997. Exon skipping and circular RNA formation in transcripts of the human cytochrome P-450 $2 \mathrm{C} 18$ gene in epidermis and of the rat androgen binding protein gene in testis. Mol Cell Biol 17: 2985-2993. 


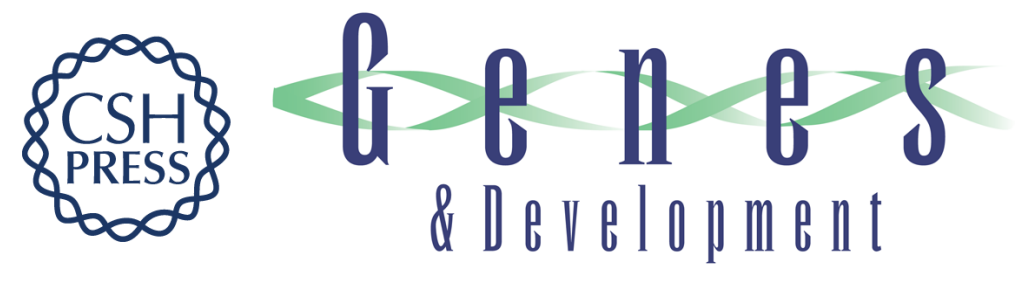

\section{Short intronic repeat sequences facilitate circular RNA production}

Dongming Liang and Jeremy E. Wilusz

Genes Dev. 2014, 28: originally published online October 3, 2014

Access the most recent version at doi:10.1101/gad.251926.114

\section{Supplemental Material \\ References \\ Creative \\ Commons \\ License}

Email Alerting
Service
http://genesdev.cshlp.org/content/suppl/2014/10/02/gad.251926.114.DC1

This article cites 40 articles, 11 of which can be accessed free at: http://genesdev.cshlp.org/content/28/20/2233.full.html\#ref-list-1

This article is distributed exclusively by Cold Spring Harbor Laboratory Press for the first six months after the full-issue publication date (see

http://genesdev.cshlp.org/site/misc/terms.xhtml). After six months, it is available under a Creative Commons License (Attribution-NonCommercial 4.0 International), as described at http://creativecommons.org/licenses/by-nc/4.0/.

Receive free email alerts when new articles cite this article - sign up in the box at the top right corner of the article or click here.

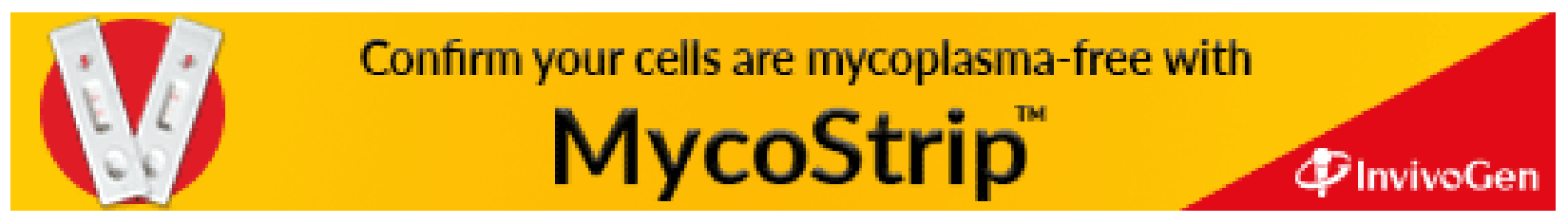

